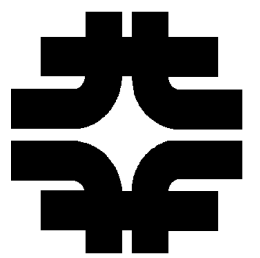

Fermilab

Proton Source Department/Beams Division

\title{
Fermilab TM 2161 \\ Observation of the Spread of the Readings in the Linac Toroids and BPMs
}

\author{
Elliott McCrory \\ Monday, October 22, 2001
}

\section{Introduction}

Measurements have been made of the spread in recent readings of the toroids and the BPMs in Linac. The intent is to estimate the accuracy of these devices.

\section{Procedure}

The procedure is first to sample the devices on a lot of beam pulses, 2000 in all for this measurement. These acquisitions occurred over the course of about an hour of Linac and Booster studies on October 12, 2001. Then, the arithmetical average and standard deviation are calculated from the data.

This table summarizes these raw results.

$\begin{array}{lcccc}\text { Device } & \text { Ave } & \text { Sigma } & \text { Units } & \text { Delta } \\ \text { L:TO1IN } & 73.43 & 1.0 & \mathrm{~mA} & 1.4 \% \\ \text { L:TO4IN } & 54.91 & 0.8 & \mathrm{~mA} & 1.5 \% \\ \text { L:TO5OUT } & 54.03 & 3.6 & \mathrm{~mA} & 6.6 \% \\ \text { L:D0TOR } & 53.57 & 1.5 & \mathrm{~mA} & 2.7 \% \\ \text { L:D1TOR } & 53.50 & 1.5 & \mathrm{~mA} & 2.9 \% \\ \text { L:D2TOR } & 53.50 & 1.5 & \mathrm{~mA} & 2.9 \% \\ \text { L:D3TOR } & 52.87 & 1.6 & \mathrm{~mA} & 3.0 \% \\ \text { L:D4TOR } & 51.79 & 1.7 & \mathrm{~mA} & 3.3 \% \\ \text { L:D5TOR } & 51.55 & 1.7 & \mathrm{~mA} & 3.4 \% \\ \text { L:D7TOR } & 52.07 & 1.7 & \mathrm{~mA} & 3.2 \% \\ \text { L:D02BPH } & -1.43 & 0.04 & \mathrm{~mm} & \\ \text { LD02BPV } & -1.40 & 0.15 & \mathrm{~mm} & \\ \text { L:D03BPH } & -2.16 & 0.09 & \mathrm{~mm} & \\ \text { L:D03BPV } & -0.09 & 0.08 & \mathrm{~mm} & \\ \text { L:D04BPH } & -0.67 & 0.05 & \mathrm{~mm} & \\ \text { L:D04BPV } & -2.33 & 0.09 & \mathrm{~mm} & \\ \text { L:D73BPH } & 4.87 & 0.32 & \mathrm{~mm} & \\ \text { L:D73BPV } & -0.28 & 0.05 & \mathrm{~mm} & \end{array}$




$\begin{array}{llll}\text { L:D74BPH } & -2.37 & 0.33 & \mathrm{~mm} \\ \text { L:D74BPV } & 0.06 & 0.15 & \mathrm{~mm}\end{array}$

In some situations, the parameter these devices is measuring actually changes, in addition to the random fluctuations targeted to measure here. If one plots the correlation between two of these parameters, it is possible to extract a deviation that is closer to the actual statistical fluctuation.
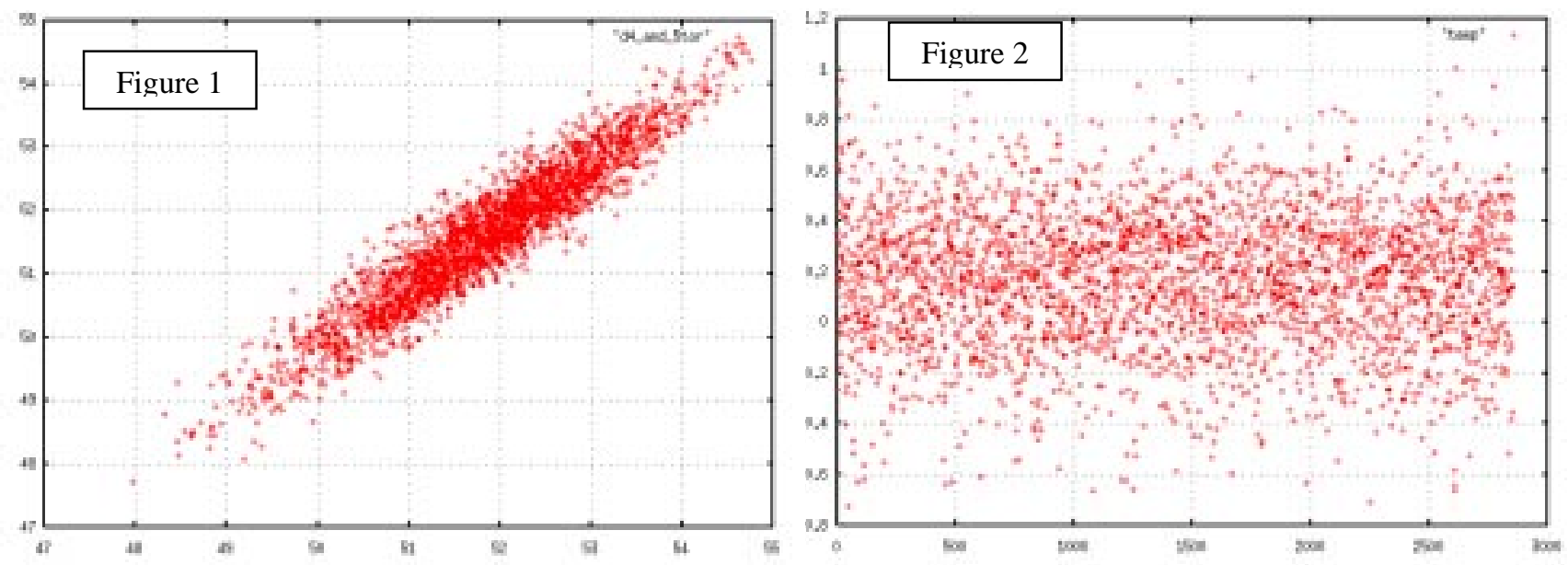

Figure 1 shows a typical (good) correlation for the toroid readings between D4TOR and D5TOR.

Figure 2 shows the distance of each of these points from the line D4TOR $=$ D5TOR -0.1 $\mathrm{mA}$. The standard deviation as calculated from the distances in Figure 2 is $0.26 \mathrm{~mA}-$ a better reading of the accuracy of the toroid readbacks.

Figure 3 is the correlation between the vertical BPMs at the input of (high-energy) module 3 and the input of module 4. Again, most of the variation is due to real motion in the beam.

Once more, if the linear correlation is removed, using the best-fit line, D04BPV $=0.548 *$ D03BPV +0.513 , one gets Figure 4 and a standard deviation of the distances from this line of $0.025 \mathrm{~mm}$.
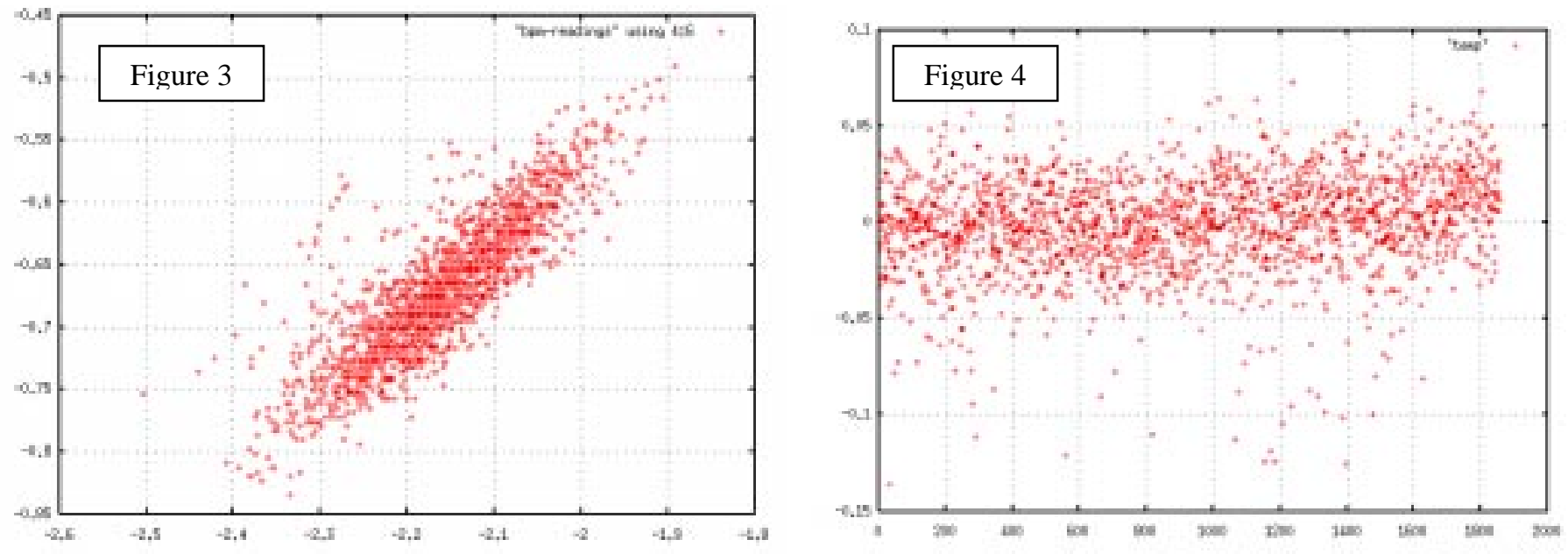


\section{Conclusion}

The readback accuracy on the high-energy toroids is about $0.26 \mathrm{~mA}$. This does not consider, completely, the relative offsets among the toroids, just the accuracy of one reading on one toroid.

Similarly, if you remove the way in which the beam moves in the beam pipe and ignore the relative offsets of the BPMs themselves, then the absolute accuracy of the reading on a BPM is 35 microns.

To summarize:

\begin{tabular}{|l|l|l|}
\hline Toroid readback Accuracy & 0.26 & $\mathrm{~mA}$ \\
\hline BPM readback Accuracy & 0.035 & $\mathrm{~mm}$ \\
\hline
\end{tabular}

